\title{
Infantile Acute Generalized Pustular Psoriasis: A Case Report
}

\author{
Gallo L, Zagaria O, Patrì A*, De Fata Salvatores G, Megna M and Balato N
}

Section of Dermatology, Department of Clinical Medicine and Surgery, University of Naples Federico II, Naples, Italy

*Corresponding author: Patrì A, MD, Section of Dermatology, Department of Clinical Medicine and Surgery, University of Naples Federico II, Via Pansini 5, Naples 80131, Italy, Fax: +39 0817462442, Tel: +39 0817462457 , E-mail: patriangela.ap@gmail.com

Citation: Gallo L, Zagaria O, Patrì A, De Fata Salvatores G, Megna M, et al. (2017) Infantile Acute Generalized Pustular Psoriasis: A Case Report. J Case Rep Stud 5(6): 603

Received Date: October 03, 2017 Accepted Date: December 27, 2017 Published Date: December 29, 2017

\begin{abstract}
Generalized pustular psoriasis (GPP) is an acute and uncommon form of psoriasis, representing about 3\% of all psoriasis cases. Triggers may include streptococcical infections, emotional stress, vaccinations and exposure to medications such as penicillin. Systemic therapy is often necessary for GPP, since it may rapidly evolve, leading to significant and potentially fatal complications. Here, we describe the case of a 5-year-old male presenting with diffuse erythema, confluent scaly plaques and painful pustules localized on the trunk and limbs. Systemic manifestations such as fever, general lymphadenopathy and arthritis were also observed. To date, no standardized treatment guideline exists for infantile acute generalized pustular psoriasis. Our patient responded successfully to cyclosporine treatment within 4 weeks without any side effects. Thus, cyclosporine could be considered a useful agent in cases of pediatric GPP, keeping in mind its side effect profile in child population.
\end{abstract}

Keywords: Pustular psoriasis; Childhood; Treatment; Cyclosporine

\section{Introduction}

Psoriasis is a chronic, immune-mediated, inflammatory disease characterized by keratinocytes hyper proliferation, affecting 2-4\% of the general population [1]. Two incidence peaks have been suggested: one before the age of 20 and another in adulthood. However, it can start at any age with $10 \%$ of cases developing before 10 and $2 \%$ prior to 2 years, with an equal gender distribution [2]. Plaque and guttate psoriasis are the most common psoriasis subtypes affecting childhood whereas other forms such as generalized pustular psoriasis (GPP) by von Zumbusch or erythrodermic one are very uncommon [3]. GPP is a rare and acute form of psoriasis, representing about 3\% of all psoriasis cases [2]. Triggers may include streptococcical infections, emotional stress, vaccinations and exposure to medications such as penicillin [3]. GPP usually arises abruptly with systemic symptoms (high fever and severe malaise) and diffuse erythemato-edematous or desquamative patches, followed by yellow-colored sterile non follicular pustules. The oral mucosa can be involved with geographic tongue and fissures. In the acute pustular phase, skin lesions can be painful whereas in a later phase, they tend to resolve with concurrent generalized desquamation [4]. Systemic therapy is often necessary for GPP since it may rapidly evolve, leading to significant complications such as secondary bacterial infection, cardiorespiratory failure, alterations of blood count, protein and electrolyte imbalance, CRP increase, renal and liver impairment [3].

\section{Case report}

A 5-year-old male presented with diffuse erythema, confluent scaly plaques and painful pustules localized on the trunk and limbs, with a particular involvement of flexural folds (Figure 1). Lesions had started on the trunk, involving the most of the body surface within 3 days. Systemic manifestations, namely fever, general lymphadenopathy and arthritis were observed. The patient had a positive family history for psoriasis. The following blood tests were performed: complete blood count with differential, liver enzymes, serum proteins, serum creatinine, glycemia, lipid determination, erythrocyte sedimentation rate (ESR), C reactive protein, rheumatoid factor. They were all within normal ranges, except for white blood cell count $\left(2.5 \mathrm{x} 10^{4} \mu \mathrm{l}\right.$ r.n; normal values 4.5-11.0 x 10 $\mu \mathrm{l})$ and ESR (15 mm/hr; normal values 3-13 mm/hr). Serological tests for cytomegalovirus, Epstein-Barr and herpes simplex virus were negative as well as pharyngeal swab. A $3 \mathrm{~mm}$ skin biopsy was taken from the left hip. Histological examination with hematoxylin-eosin showed acanthosis, parakeratosis in epidermis, exocytosis of neutrophils and small pustule formation in the stratum corneum as well as moderate perivascular inflammatory infiltrate in the superficial dermis. 


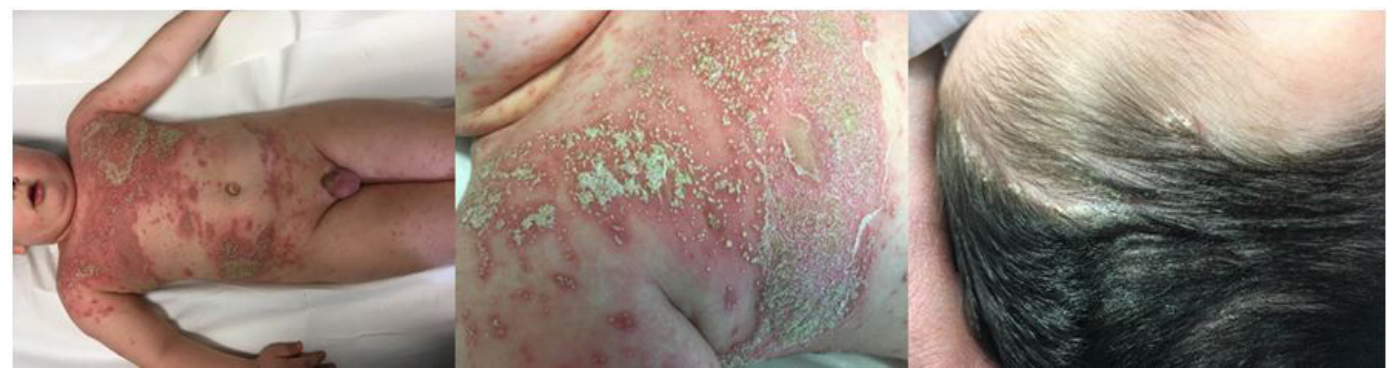

Figure 1: A 5-year-old male presenting with diffuse erythema, confluent scaly plaques and painful pustules localized on the trunk and limbs

Based on clinical and histopatological findings, the diagnosis of pustular psoriasis was made (PASI 27.4; BSA 60\%). Treatment with cyclosporine $(100 \mathrm{mg} /$ daily - $2 \mathrm{mg} / \mathrm{kg} /$ day) was commenced and continued for one month, achieving a significant clinical improvement (PASI 10.4; BSA 25\%). Then, cyclosporine dosage was reduced to $1 \mathrm{mg} / \mathrm{kg} / \mathrm{day}$ and is still ongoing. To date, after a three months follow up, no recurrence of GPP has been observed. Figures 2 and 3 show respectively patient clinical improvement after 20 and 45 days of treatment with cyclosporine.

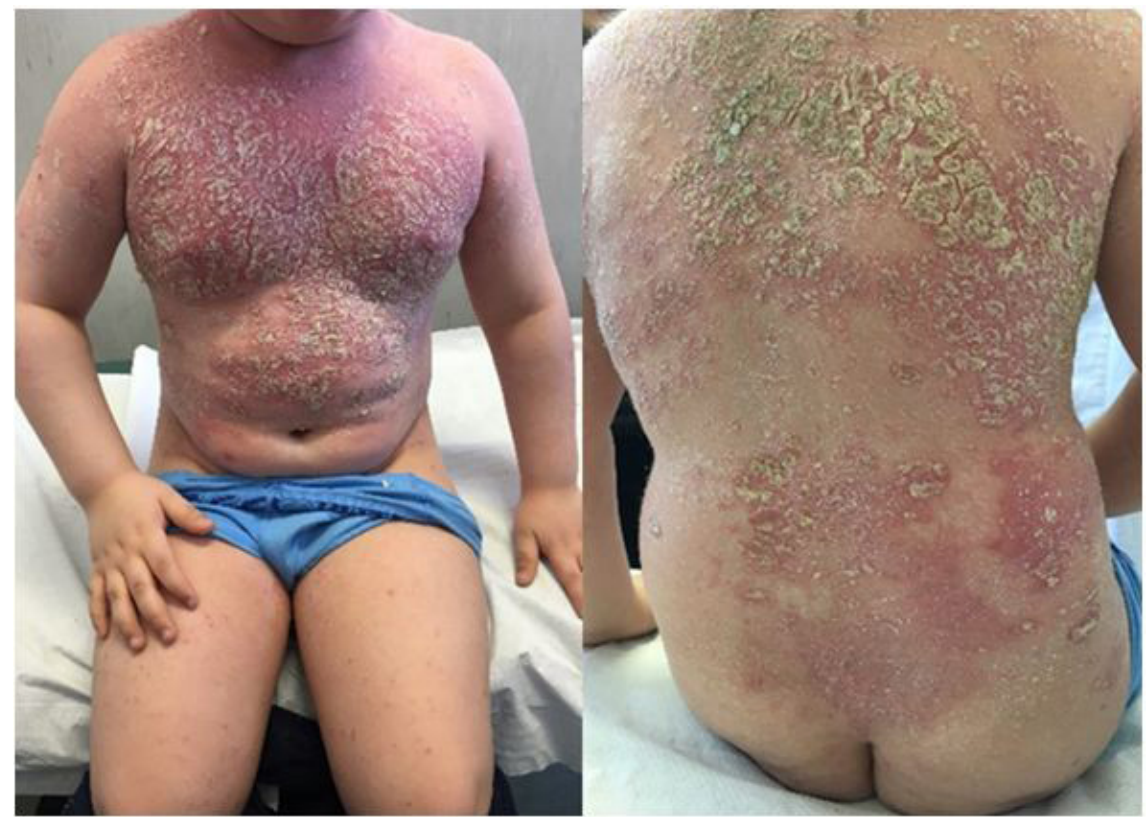

Figure 2: Patient clinical improvement after 20 days of treatment with cyclosporine

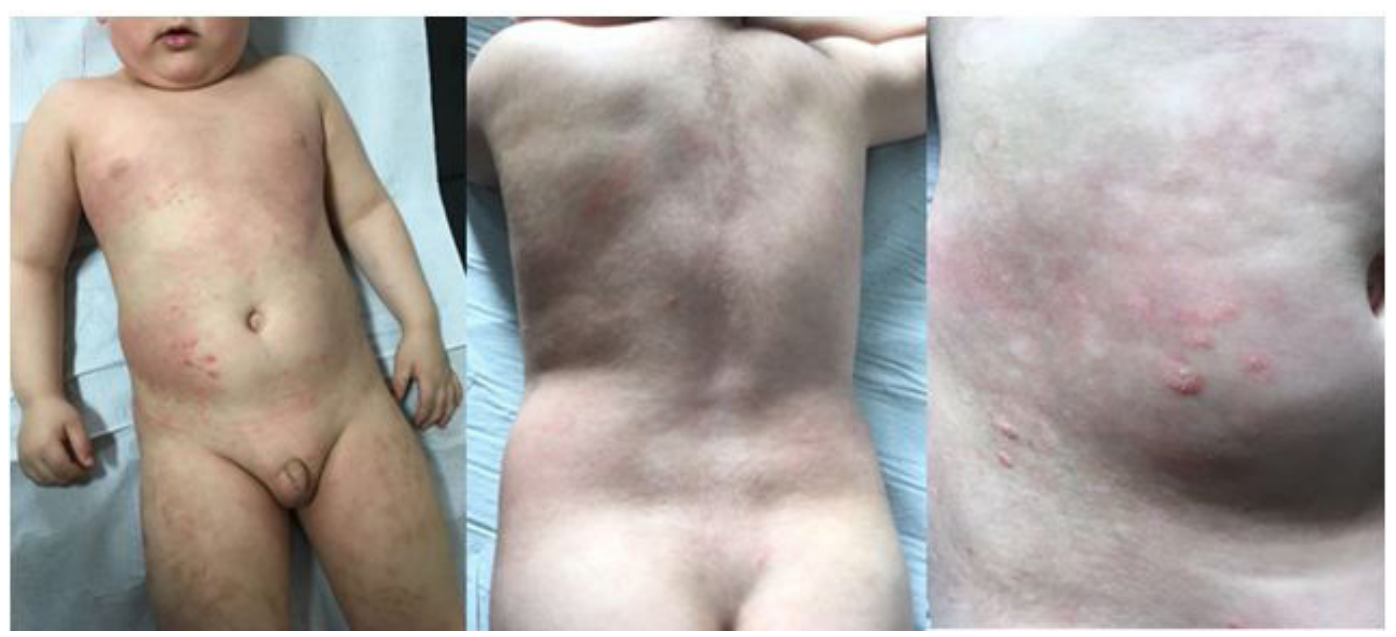

Figure 3: Almost complete remission after 45 days of treatment with cyclosporine

\section{Discussion}

Pustular psoriasis is an uncommon form of psoriasis including localized and generalized subtypes. Localized forms can be divided into palmoplantar pustulosis and acrodermatitis continua of Hallopeau (ACH) whereas generalized forms are classified in acute 
generalized pustular psoriasis (GPP) of von Zumbusch, impetigo herpetiformis, annular pustular psoriasis, juvenile and infantile pustular psoriasis [4].

GPP is a severe psoriasis subtype; whose pathogenesis is still unclear [5]. The characteristic lesion is defined as primary, sterile, macroscopically visible pustule on non-acral skin (excluding cases where pustulation is restricted to psoriatic plaques). GPP can occur with or without systemic inflammation and can either be a relapsing (> 1 episode) or persistent (> 3 months) condition [6].

In children, differential diagnosis must be performed with several conditions such as subcorneal pustulosis, IgA pemphigus and acute generalized exanthematous pustulosis (AGEP).

To date, no standardized treatment guideline exists for infantile acute generalized pustular psoriasis.

According to case reports and case series, acitretin seems to be the treatment of choice for this condition [7]. However, good results have also been observed with cyclosporine [8], methotrexate or biologic agents such as etanercept, adalimumab and ustekinumab for recalcitrant cases [3,9-13]. Cyclosporine is an immunosuppressive drug which acts by inhibiting interleukin-2 and $\mathrm{T}$ cells functions [2]. Renal toxicity, hypertension and immunosuppression often limit its use; thus, laboratory and blood pressure monitoring before and during treatment is essential [2]. Psoriasis relapse may occur after cyclosporine discontinuation, although timing and rate of relapse can vary considerably among individuals [14]. Our pediatric patient responded to cyclosporine treatment within 4 weeks without any side effects. According to Kilic et al. [14], low doses of cyclosporine for short periods might be used for the treatment of severe episodes of infantile pustular psoriasis.

\section{Conclusion}

In conclusion, despite the absence of standardized guidelines, cyclosporine should be considered a useful agent in cases of pediatric GPP, keeping in mind its efficacy and side effect profile in child population.

\section{References}

1. Di Costanzo L, Fattorusso V, Mozzillo E, Patrì A, Di Caprio R, et al. (2017) Psoriasis in children with type 1 diabetes: a new comorbidity to be considered? Acta Diabetol 54: 803-4.

2. Megna M, Napolitano M, Balato A, Scalvenzi M, Cirillo T, et al. (2015) Psoriasis in children: a review. Curr Pediatr Rev 11: 10-26.

3. Saikaly SK, Mattes M (2016) Biologics and pediatric generalized pustular psoriasis: an emerging therapeutic trend. Cureus 8: e652.

4. van de Kerkhof PCM (2003) Textbook of Psoriasis (2 ${ }^{\text {nd }}$ Edn) Wiley-Blackwell, USA.

5. Popadic S, Nikolic M (2014) Pustular psoriasis in childhood and adolescence: a 20-year single-center experience. Pediatr Dermatol 31: 575-9.

6. Navarini AA, Burden AD, Capon F, Mrowietz U, Puig L, et al. (2017) European Consensus Statement on Phenotypes of Pustular Psoriasis. J Eur Acad Dermatol Venereol 10.1111/jdv.14386.

7. Pilkington T, Brogden RN (1992) Acitretin: a review of its pharmacology and therapeutic use. Drugs 43: 597-627.

8. Dogra S, Mahajan R, Narang T, Handa S (2017) Systemic cyclosporine treatment in severe childhood psoriasis: a retrospective chart review. J Dermatolog Treat 28: $18-20$.

9. Pan J, Qiu L, Xiao T, Chen HD (2016) Juvenile generalized pustular psoriasis with IL36RN mutation treated with short-term infliximab. Dermatol Ther 29: 164-7.

10. Skrabl-Baumgartner A, Weger W, Salmhofer W, Jahnel J (2015) Childhood generalized pustular psoriasis: long time remission with combined infliximab and methotrexate treatment. Pediatr Dermatol 32: e13-4.

11. Posso-De Los Rios CJ, Pope E, Lara-Corrales I (2014) A systematic review of systemic medications for pustular psoriasis in pediatrics. Pediatr Dermatol 31: 430-9.

12. Fialová J, Vojáčková N, Vaňousová D, Hercogová J (2014) Juvenile generalized pustular psoriasis treated with etanercept. Dermatol Ther 27: 105-8.

13. Robinson A, Van Voorhees AS, Hsu S, Korman NJ, Lebwohl MG, et al. (2012) Treatment of pustular psoriasis: from the Medical Board of the National Psoriasis Foundation. J Am Acad Dermatol 67: 279-88.

14. Kiliç SS, Hacimustafaoğlu M, Celebi S, Karadeniz A, Ildirim I (2001) Low dose cyclosporin A treatment in generalized pustular psoriasis. Pediatr Dermatol 18: $246-8$. 


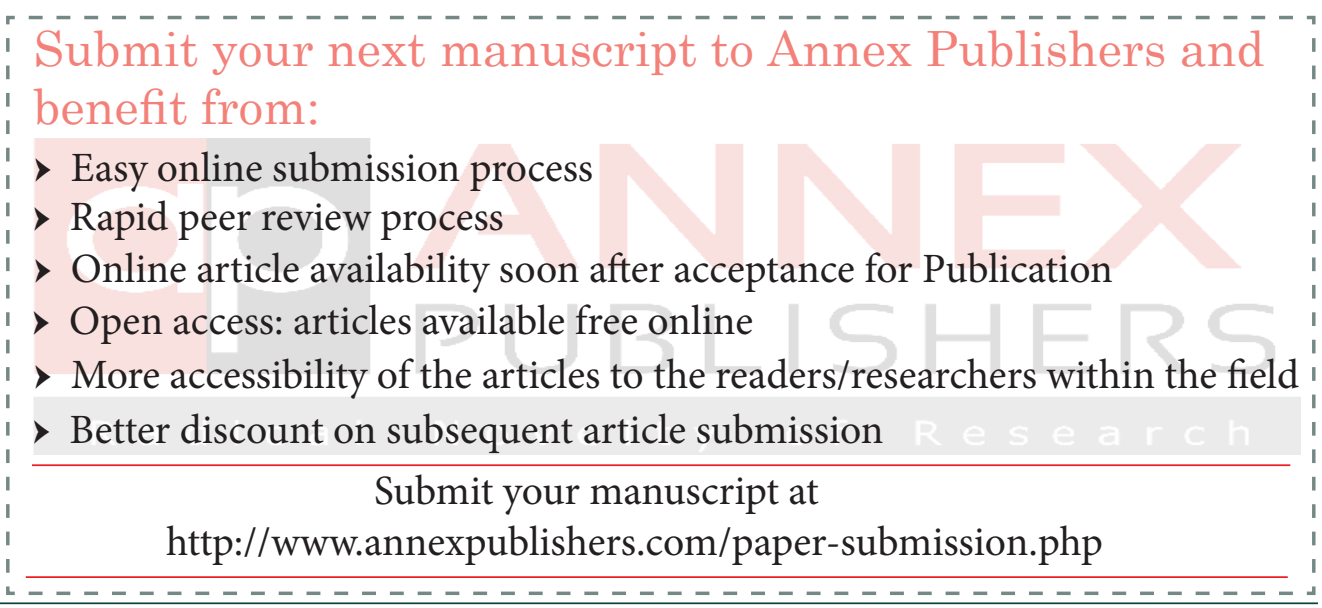

